\title{
Identification of an immunogenic protein of Giardia lamblia using monoclonal antibodies generated from infected mice
}

\author{
Jael Quintero', Diana Carolina Figueroa ${ }^{2}$, Rafael Barcelo ${ }^{3}$, Linda Breci ${ }^{4}$, Humberto Astiazaran-Garcia ${ }^{5}$, \\ Lucila Rascon ${ }^{3}$, Ramon Robles-Zepeda ${ }^{3}$, Adriana Garibay-Escobar ${ }^{3}$, Enrique Velazquez-Contreras ${ }^{1}$, \\ Gloria Leon Avila ${ }^{6}$, Jose Manuel Hernandez-Hernandez ${ }^{2}$, Carlos Velazquez ${ }^{3 /+}$
}

\author{
'Department of Polymers and Materials ${ }^{3}$ Department of Chemistry-Biology, University of Sonora, Hermosillo, Sonora, México \\ 2Department of Cellular Biology, Centro de Investigación y de Estudios Avanzados, México DF, México \\ ${ }^{4}$ Department of Chemistry and Biochemistry, University of Arizona, Tucson, Arizona, USA \\ ${ }^{5}$ Department of Nutrition and Metabolism, Centro de Investigación en Alimentación y Desarrollo AC, Hermosillo, Sonora, México \\ ${ }^{6}$ Department of Zoology, Escuela Nacional de Ciencias Biológicas, México DF, México
}

The humoral immune response plays an important role in the clearance of Giardia lamblia. However, our knowledge about the specific antigens of $\mathrm{G}$. lamblia that induce a protective immune response is limited. The purpose of this study was to identify and characterise the immunogenic proteins of $\mathrm{G}$. lamblia in a mouse model. We generated monoclonal antibodies (moAbs) specific to G. lamblia (1B10, 2C9.D11, 3C10.E5, 3D10, 5G8.B5, 5F4, 4C7, 3C5 and 3C6) by fusing splenocytes derived from infected mice. Most of these moAbs recognised a band of $\pm 71 \mathrm{kDa}(5 \mathrm{G} 8$ protein) and this protein was also recognised by serum from the infected mice. We found that the moAbs recognised conformational epitopes of the $5 G 8$ protein and that this antigen is expressed on the cell surface and inside trophozoites. Additionally, antibodies specific to the $5 G 8$ protein induced strong agglutination (> 70-90\%) of trophozoites. We have thus identified a highly immunogenic antigen of $\mathrm{G}$. lamblia that is recognised by the immune system of infected mice. In summary, this study describes the identification and partial characterisation of an immunogenic protein of G. lamblia. Additionally, we generated a panel of moAbs specific for this protein that will be useful for the biochemical and immunological characterisation of this immunologically interesting Giardia molecule.

Key words: Giardia lamblia - immunogenic antigen - monoclonal antibody

Giardia lamblia is the causative agent of giardiasis in humans and several animal species, colonising the small intestinal lumen (Adam 2001). This parasite is widely distributed worldwide (Lengerich et al. 1994). The signs and symptoms of giardiasis include diarrhoea, abdominal pain, flatulence, weight loss and malabsorption of nutrients. In most cases, the infection remains asymptomatic (Adam 2001, Eckmann \& Gillin 2001). The immunological mechanisms involved in clearance of Giardia infections are not fully elucidated. In immunocompetent patients, the infection is self-limiting, which indicates the presence of effective host defence mechanisms against this intestinal parasite (Faubert 2000, Eckmann 2003). The humoral and cellular immune responses play an important role in the control of Giardia infections (Faubert 2000, Singer \& Nash 2000, Roxstrom-Lindquist et al. 2006). However, our knowledge about the immunogenic antigens of G. lamblia is limited. Several antigenic proteins of $G$. lamblia have been described, including variable surface proteins (VSPs), cyst wall proteins (CWPs), giardins, tubulins, arginine deaminase, ornithine carbamoyl transferase and enolase, among others (Luján et

doi: 10.1590/0074-0276108052013013

Financial support: CONACYT (CB-2010-01/155224)

+ Corresponding author: velaz@guayacan.uson.mx

Received 4 December 2012

Accepted 1 March 2013 al. 1995, Langford et al. 2002, Abdul-Wahid \& Faubert 2004, Davids et al. 2006). Several of these molecules are recognised by the systemic and secretory antibody responses of infected humans and mice (Palm et al. 2003, Abdul-Wahid \& Faubert 2004, Téllez et al. 2005, Davids et al. 2006). The proteins $\alpha-1$ giardin and CWP-2 provide marked protection against G. lamblia infection (Larocque et al. 2003, Jenikova et al. 2011).

The purpose of this study was to identify and characterise the immunogenic proteins of $G$. lamblia in a mouse model by generating monoclonal antibodies (moAbs) specific to $G$. lamblia by using splenocytes derived from infected mice. Those moAbs will help to further characterise the Giardia antigens that are recognised during infection.

\section{MATERIALS AND METHODS}

Mice - $\mathrm{C} 3 \mathrm{H} / \mathrm{HeJ}$ mice were purchased from The Jackson Laboratory (Bar Harbor, ME, USA). This strain of mouse is susceptible to infection with the G. lamblia clone GS/M-83-H7 (Byrd et al. 1994).

G. lamblia culture - G. lamblia trophozoites [clone GS/M-83-H7 (ATCC 50581)] were obtained from the American Type Culture Collection. Axenic G. lamblia cultures were maintained in the TYI-S-33 medium, which was supplemented with newborn calf serum (NBCS) and antibiotics at $37^{\circ} \mathrm{C}$.

G. lamblia protein extract - G. lamblia soluble extract proteins were obtained using the method described by Gottstein et al. (1990), with slight modifications. 
Confluent cultures of G. lamblia trophozoites were harvested during log-phase growth and were incubated on ice for $15 \mathrm{~min}$. The harvested trophozoites were washed three times with sterile phosphate buffered saline (PBS). Then, the trophozoites were resuspended in $1.5 \mathrm{~mL}$ of PBS and frozen (liquid nitrogen) and thawed [room temperature (RT)] three times in the presence of a protease inhibitor cocktail [23 mM 4-(2-aminoethyl) benzenesulphonyl fluoride, $0.3 \mathrm{mM}$ pepstatin A, $0.3 \mathrm{mM}$ E- 64,2 $\mathrm{mM}$ bestatin and $100 \mathrm{mM}$ sodium ethylenediamine tetraacetic acid (Sigma, St. Louis, MO, USA)].

The G. lamblia lysate was sonicated for 2 min (30 cycles) (Brandon Sonifier 250, Shelton, CT, USA). Cell debris was removed by centrifugation $(10,000 \mathrm{~g}$ for 30 $\mathrm{min}$ ). The protein concentration of the soluble antigen preparation was determined by the Bradford method (Bio-Rad, Hercules, CA, USA).

Generation of Giardia specific B-cell hybridomas B-cell hybridomas were generated from splenocytes of G. lamblia-infected $\mathrm{C} 3 \mathrm{H} / \mathrm{HeJ}$ mice. Eight-14-week-old male $\mathrm{C} 3 \mathrm{H} / \mathrm{HeJ}$ mice were infected and re-infected with $5 \times 10^{6}$ G. lamblia trophozoites (GS/M-83-H7) using a sterile animal-feeding needle for peroral inoculation. The G. lamblia inoculum was prepared by washing in vitro-cultivated trophozoites three times with ice-cold sterile PBS and resuspending them in $200 \mu \mathrm{L}$ of sterile PBS. Primary infection occurred on day 0 , while the second and third challenges took place on days 42 and 84 , respectively. The infected mice were bled from the tail vein every week and the serum was recovered and stored at $-80^{\circ} \mathrm{C}$. Infected mice were sacrificed by cervical dislocation five days after the last infection. Splenocytes were fused with the myeloma cell line P3 x 63.Ag8 using polyethylene glycol 1500 (Roche, Indianapolis, IN). moAbs specific to G. lamblia proteins were selected on the basis of their antigen recognition and were subcloned by limiting dilution (Velazquez et al. 2005).

Western blotting assays - Soluble G. lamblia proteins were separated by sodium dodecyl sulfate polyacrylamide gel electrophoresis (SDS-PAGE) (12\%) under modified reducing conditions. G. lamblia protein extract (500 $\mu \mathrm{g})$ was mixed with an equal volume of modified $2 \mathrm{X}$ SDS-PAGE sample buffer $(0.2 \%$ SDS, $0.2 \%$ 2-mercaptoethanol). These modifications in the SDS-PAGE sample buffer were performed after preliminary experiments indicated that the antigen-recognition of the moAb $5 \mathrm{G} 8$. B5 was sensitive to the standard concentration of SDS and 2-mercaptoethanol (Velazquez et al. 2005). The proteins were electrotransferred to a nitrocellulose membrane for $20 \mathrm{~min}$ using a semi-dry blotting system (Owl HEP-1, Thermo Scientific, Rockford, IL, USA) with a 120-mAmp current. The nitrocellulose membranes were blocked with PBS containing 5\% fat-free dry milk and 1\% bovine serum albumin (BSA) for $1 \mathrm{~h}$ at RT. The blocked membranes were then incubated for $1 \mathrm{~h}$ at RT either with mouse serum from pre-infected mice, infected and reinfected mice (diluted 1:25) or with several moAbs [antiVSP H7 ascites serum (moAb G10/4), anti-5G8 protein (moAb 5G8.B5) and control antibodies (isotype control antibody) (IgG2b, from Sigma, St. Louis MO, USA) and 40F (IgG2a)]. All moAbs were tested at $5 \mu \mathrm{g} / \mathrm{mL}$. After five washes with PBS, the membranes were incubated with horseradish peroxidase-conjugated goat antimouse IgG (diluted 1:7500 with PBS-0.1\% BSA) (Sigma, St. Louis MO, USA) for $1 \mathrm{~h}$ at RT. The membranes were washed and developed using a SuperSignal West Pico Chemoluminescent Substrate kit (Thermo Scientific, Rockford, IL, USA).

Flow cytometric analysis of G. lamblia trophozoites - For cell surface staining, trophozoites were washed twice with ice-cold PBS. Then, the cells were incubated with the moAb 5G8.B5 (IgG2b) or the isotype control antibody (IgG2b) at $5 \mu \mathrm{g} / \mathrm{mL}$ in D5F- $0.05 \% \mathrm{NaN}_{3}$ for 1 $\mathrm{h}$ at $4^{\circ} \mathrm{C}$. After three washes with cold PBS, the trophozoites were incubated with fluorescein isothiocyanate (FITC)-conjugated goat anti-mouse IgG (diluted 1:200 with $\mathrm{D} 5 \mathrm{~F}-0.05 \% \mathrm{NaN}_{3}$ ) (Sigma, St. Louis, MO, USA) for $1 \mathrm{~h}$ at $4^{\circ} \mathrm{C}$. Then, the trophozoites were washed three times with cold PBS and fixed for $15 \mathrm{~min}$ at RT with $1 \%$ paraformaldehyde (PFA) (Sigma, St. Louis MO, USA) in Dulbecco's Modified Eagle's Medium (Sigma, St. Louis MO, USA) supplemented with $5 \%$ heat-inactivated foetal bovine serum (FBS) containing $0.05 \% \mathrm{NaN}_{3}$ (D5F$0.05 \% \mathrm{NaN}_{3}$ ). At least 10,000 trophozoites were acquired using fluorescence-activated cell sorting (FACS) (Canto II FACS, Becton Dickinson, CA, USA). For intracellular staining of the trophozoites, the Giardia cells were fixed for 15 min with $1 \%$ PFA at RT. After two washes with ice-cold PBS, the trophozoites were permeabilised for 5 min at RT with $0.1 \%$ triton X-100 (Sigma, St. Louis MO, USA). The permeabilised cells were then stained as described above. The fixation procedure did not alter the antigenicity of the Giardia proteins.

Confocal microscopy analysis - G. lamblia trophozoites were obtained from confluent cultures and washed three times with ice-cold sterile PBS. The trophozoites were fixed for $15 \mathrm{~min}$ in $3.7 \%$ formaldehyde. The dry glass slides were placed in PBS containing $1 \%$ milk and $5 \%$ FBS (GIBCO) for $1 \mathrm{~h}$. After three washes with icecold PBS, the slides were stained (cell-surface staining or intracellular staining) in a humidity chamber for $1 \mathrm{~h}$ at 5 $\mu \mathrm{g} / \mathrm{mL}$, using the moAb 5G8.B5 (IgG2b) or the isotype control antibody (IgG2b). After two washes with cold PBS, the slides were incubated for $1 \mathrm{~h}$ with FITC-conjugated goat anti-mouse IgG (diluted 1:100) (Sigma, St. Louis, MO, USA). The stained slides were preserved with mounting media (Vectashield) and analysed by confocal microscopy using a TCP-SP2 Confocal Laser Scanning microscope (Leica Microsystems, Heidelberg, Germany). The samples were observed through a 100X plan apochromatic oil immersion lens (NA: 1.32) and analysed with Leica Confocal Software (Leica LCS 2.0 Build 0871).

Immunoadsorption assay - Each well of a six-well plate (Corning, USA) was treated with $1 \mathrm{~mL}$ of moAb 5G8.B5 or control antibody $(25 \mu \mathrm{g} / \mathrm{mL})$ in sterile PBS for $1 \mathrm{~h}$ at $37^{\circ} \mathrm{C}$. Then, the wells were blocked with sterile PBS containing $1 \% \mathrm{BSA}$ for $1 \mathrm{~h}$ at $37^{\circ} \mathrm{C}$. After five washes with sterile cold PBS, $2 \times 10^{6}$ G. lamblia trophozoites (pre-incubated at $4^{\circ} \mathrm{C}$ ) suspended in $1 \mathrm{~mL}$ of 
PBS-1\% BSA were added to each well and incubated for $20 \mathrm{~min}$ at $4^{\circ} \mathrm{C}$. After five washes with sterile cold PBS, $2 \mathrm{~mL}$ of TYI-S-33 medium (supplemented with $10 \%$ of NBCS) was added to the wells and incubated for $10 \mathrm{~min}$ at $4^{\circ} \mathrm{C}$. The adherent cells were harvested and cultivated in TYI-S-33 medium supplemented with 10\% NBCS.

Agglutination assay - Two million G. lamblia trophozoites were incubated at $4^{\circ} \mathrm{C}$ for $1 \mathrm{~h}$ with several concentrations of the moAb 5G8.B5 or the isotype control antibody $(0,0.6,1.2,2.5,5$ or $10 \mu \mathrm{g} / \mathrm{mL})$ in ice-cold PBS in each well of a six-well plate (Corning, USA). Agglutination of the trophozoites was assessed by microscopy (Rivero et al. 2010).

Statistical analysis - The data were analysed by the Kruskal-Wallis and Tukey methods (Statistical Package for the Social Sciences 2002). Statistical significance was accepted when $\mathrm{p}<0.05$.

\section{RESULTS}

Generation of B-cell hybridomas specific for $G$. lamblia proteins - To identify and characterise the immunogenic proteins from G. lamblia, we generated Bcell hybridomas specific for G. lamblia (GS/M-83-H7) antigens by fusing splenocytes from infected mice with the myeloma cell line P3 X 63.Ag8. We generated nine moAbs that were specific for Giardia proteins (1B10, 2C9.D11, 3C10.E5, 3D10, 5G8.B5, 5F4, 4C7, 3C5 and 3C6) (Fig. 1). All of the moAbs were of the IgG2b isotype (except for the moAb 1B10, which was IgG2a) and recognised a band with a relative molecular mass of \pm $71 \mathrm{kDa}$. This immunogenic band was also recognised by serum from infected and re-infected mice (Fig. 2). To further characterise this immunogenic protein, we selected the B-cell hybridoma 5G8.B5 for its ability to produce large amounts of moAbs. To evaluate the antigen recognition by the moAb 5G8.B5, western blotting

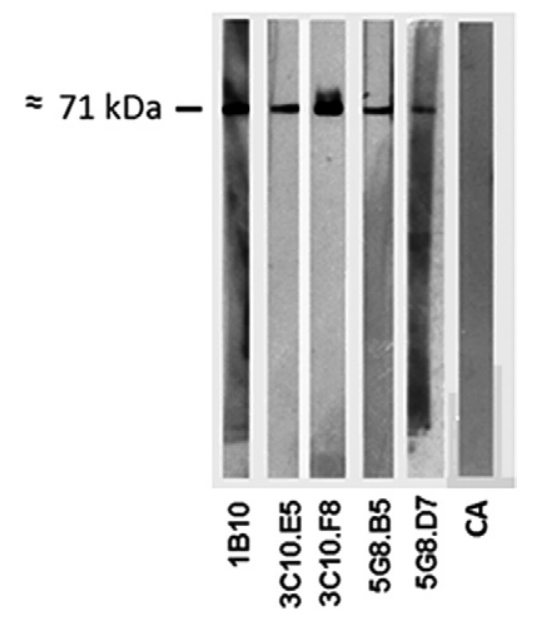

Fig. 1: antigen recognition by monoclonal antibodies (moAbs) specific for Giardia lamblia proteins. Western Blot analysis of G. lamblia specific moAbs (1B10, 3C10.E5, 3C10.F8, 5G8.B5 and 5G8.D7). Antigen recognition of some the moAbs generated. The most of them recognised a band of $\pm 71 \mathrm{kDa}$. CA: control antibody. analysis was performed. However, when the moAb recognition was evaluated, considerable difficulty was encountered in optimising the western blotting conditions. Eventually, results were obtained by reducing the SDS and 2-mercaptoethanol concentrations in the buffer prior to SDS-PAGE (see Materials and Methods). These observations indicated that antigen recognition by the $5 \mathrm{G} 8 . \mathrm{B} 5$ antibody was sensitive to the SDS and 2-mercaptoethanol conditions, suggesting that this moAb recognised a conformational epitope of the $\pm 71 \mathrm{kDa}$ protein.

$5 G 8$ protein expression in $G$. lamblia trophozoites - The relative level and distribution of expression of the $5 \mathrm{G} 8$ protein in G. lamblia trophozoites (GS/M-83-H7) was explored by performing flow cytometry analysis. Almost all permeabilised GS/M-83-H7 trophozoites $(\approx 94 \%)$ expressed the $5 \mathrm{G} 8$ protein (Fig. 3B), while only a small percentage $(\approx 2-5 \%)$ of those trophozoites expressed high levels $\left(10^{4}-10^{5} \mathrm{FI}\right.$ units) of the $5 \mathrm{G} 8$ protein on the cell surface (Fig. 3A). Confocal microscopy analysis confirmed that the $5 \mathrm{G} 8$ protein was found both at the cell surface (Fig. 4A) and inside the trophozoites (Fig. 4B). The 5G8 protein was detected as an intense and diffuse mark in all parts of trophozoites, including the ventral disc and flagella (Fig. 4A). To further characterise this immunogenic protein, we enriched the trophozoite population expressing the $5 \mathrm{G} 8$ protein on the cell surface by a panning technique using the moAb 5G8.B5. Approximately 50-70\% of the enriched cell population [5G8 (+) trophozoites] expressed the $5 \mathrm{G} 8$ protein on the cell surface.

$5 G 8$ protein is different from the VSP H7 - VSPs are immunogenic proteins that cover the cell surface of $G$. lamblia. Only one VSP is expressed on the surface of G. lamblia trophozoites at any point in time (Touz et al. 2008, Rivero et al. 2010). The G. lamblia clone GS/M-83 -H7 expresses the immunogenic VSP H7. The predicted molecular weight of this protein is $\pm 56 \mathrm{kDa}$, but the mi-

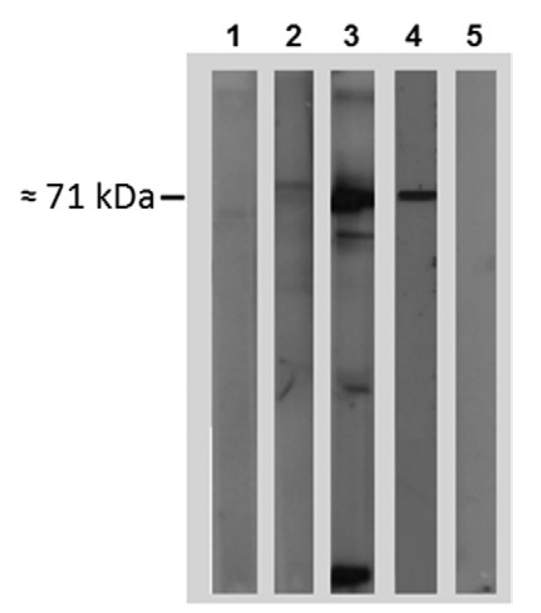

Fig. 2: the $71 \mathrm{kDa}$ Giardia lamblia protein is recognised by sera from infected and re-infected mice. 1: pre-infected serum; 2 : serum from infected mice (5th week post-infection); 3: serum from re-infected mice (2nd week post re-infection); 4: 5G8.B5 monoclonal antibody (moAb); 5: isotype control antibody (IgG2b). 
gration of this protein in as SDS-PAGE gel is considerably higher $( \pm 72 \mathrm{kDa})$. To determine whether the Giardia antigens VSP H7 and 5G8 corresponded to the same protein molecule, we evaluated the expression of these antigens on the cell surface of G. lamblia trophozoites [GS/M-83$\mathrm{H} 7$ and 5G8 (+)] by FACS analysis, using moAbs specific to the VSP H7 (G10/4 moAb) and 5G8 (5G8.B5 moAb) proteins. The antigen recognition by both moAbs was clearly different (Fig. 5), indicating that the 5G8 protein and the VSP H7 protein are not the same molecule. Additionally, confocal microscopy data showed differences in the antibody recognition pattern between the 5G8 and VSP H7 proteins (unpublished observations).

Agglutination assay - The agglutinating capacity of the moAb 5G8.B5 was evaluated in GS/M-83-H7 and 5G8 (+) trophozoite cultures. The moAb 5G8.B5 induced a strong agglutination of 5G8 (+) trophozoites at the different concentrations tested (Table). Large clumps of agglutinated cells were observed at antibody concentrations of 2.5 and $5.0 \mu \mathrm{g} / \mathrm{mL}$ (Fig. 6). In contrast, the moAb 5G8.B5 showed a lower agglutination capacity for GS/M-83-H7 trophozoites (Fig. 6, Table). The isotype-matched control antibody did not show an agglutinating capacity on Giardia cells at any of the tested antibody concentrations.

\section{DISCUSSION}

The immunological mechanisms involved in the control and clearance of Giardia are not completely understood. The humoral and cellular immune responses play an important role in Giardia infection (Faubert 2000, Singer \& Nash 2000, Roxstrom-Lindquist et al. 2006). The development of a vaccine against this infection has been hampered by a lack of knowledge about the Giardia proteins that induce a protective response during infection. In the present study, we generated moAbs specific for $G$. lamblia antigens from splenocytes derived from infected mice. These moAbs have allowed us to partially

A
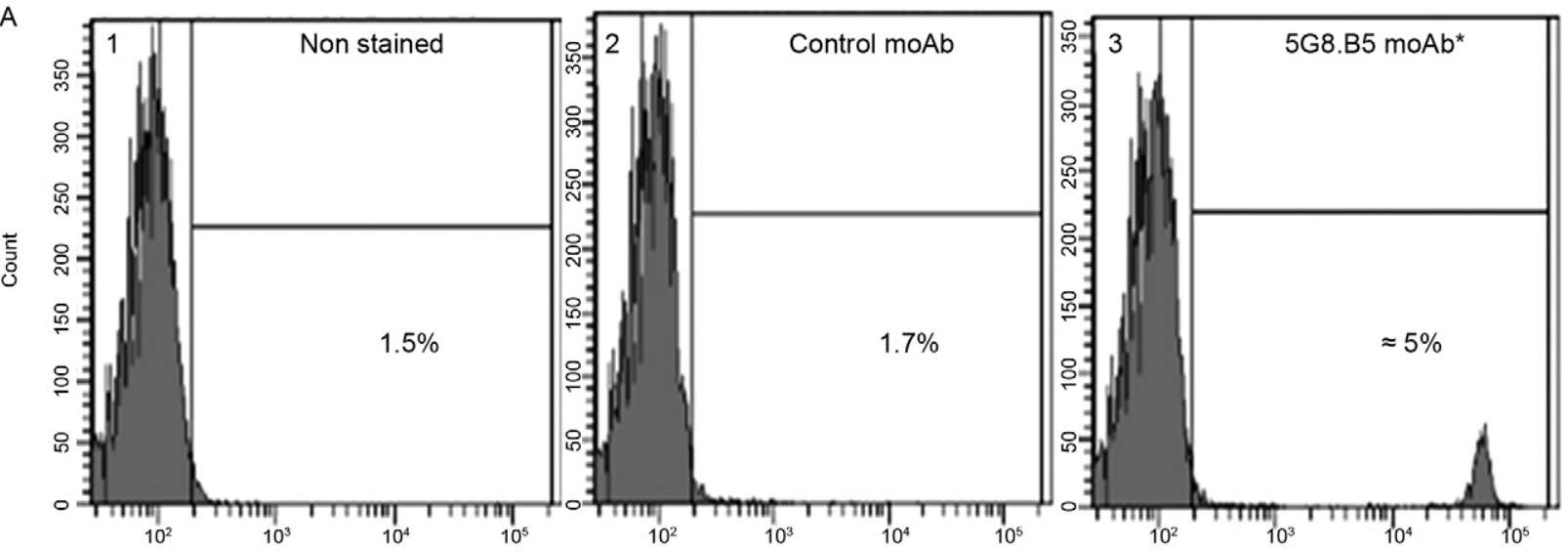

GS/M-83-H7

B

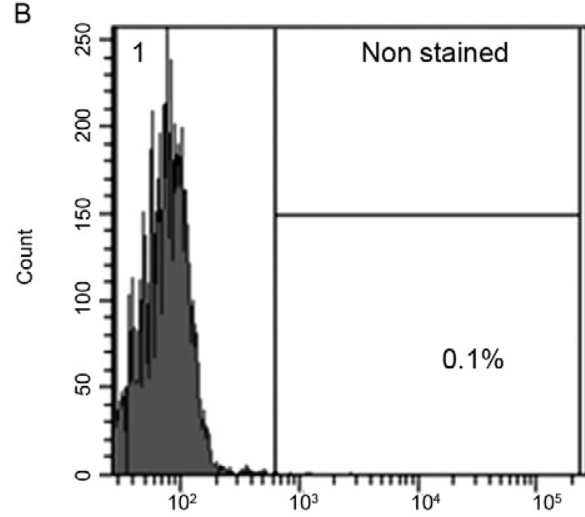

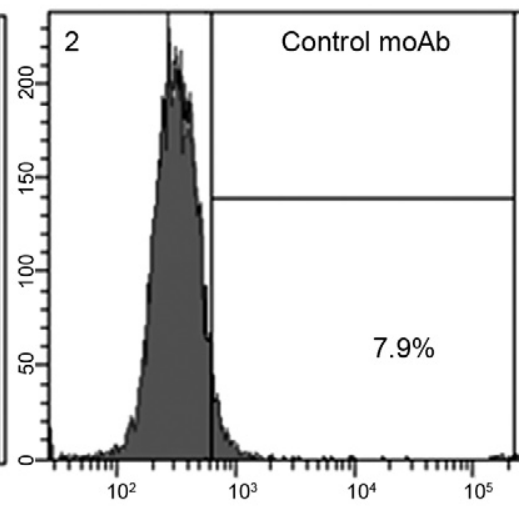

GS/M-83-H7

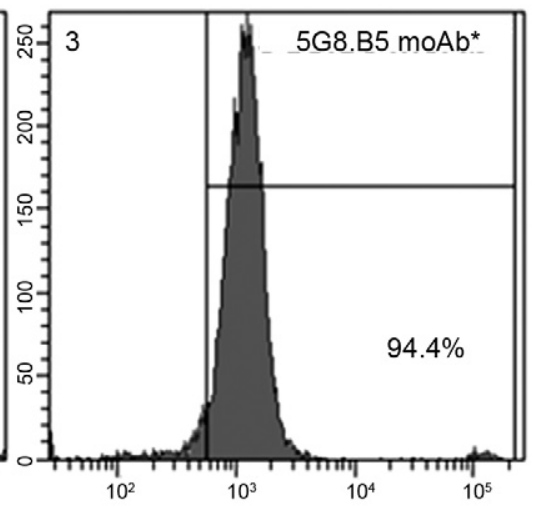

Fig. 3: $5 \mathrm{G} 8$ protein expression in Giardia lamblia trophozoites. Fluorescence-activated cell sorting analysis was performed using the 5G8.B5 monoclonal antibody (moAb) and control antibodies (isotypes IgG2b and IgG2a). A: cell surface staining of G. lamblia trophozoites (GS/M-83H7) \{1: non-stained control cells; 2: control moAb [40F (IgG2a)]; 3; 5G8.B5 moAb\}; B: inside staining of G. lamblia trophozoites (GS/M-83-H7) \{1: non-stained control cells; 2: control moAb [40F (IgG2a)]; 3: 5G8.B5 moAb\}. Statistical significance was accepted when $\mathrm{p}<0.05$ from control antibody (marked with asterisks). 
A Surface staining

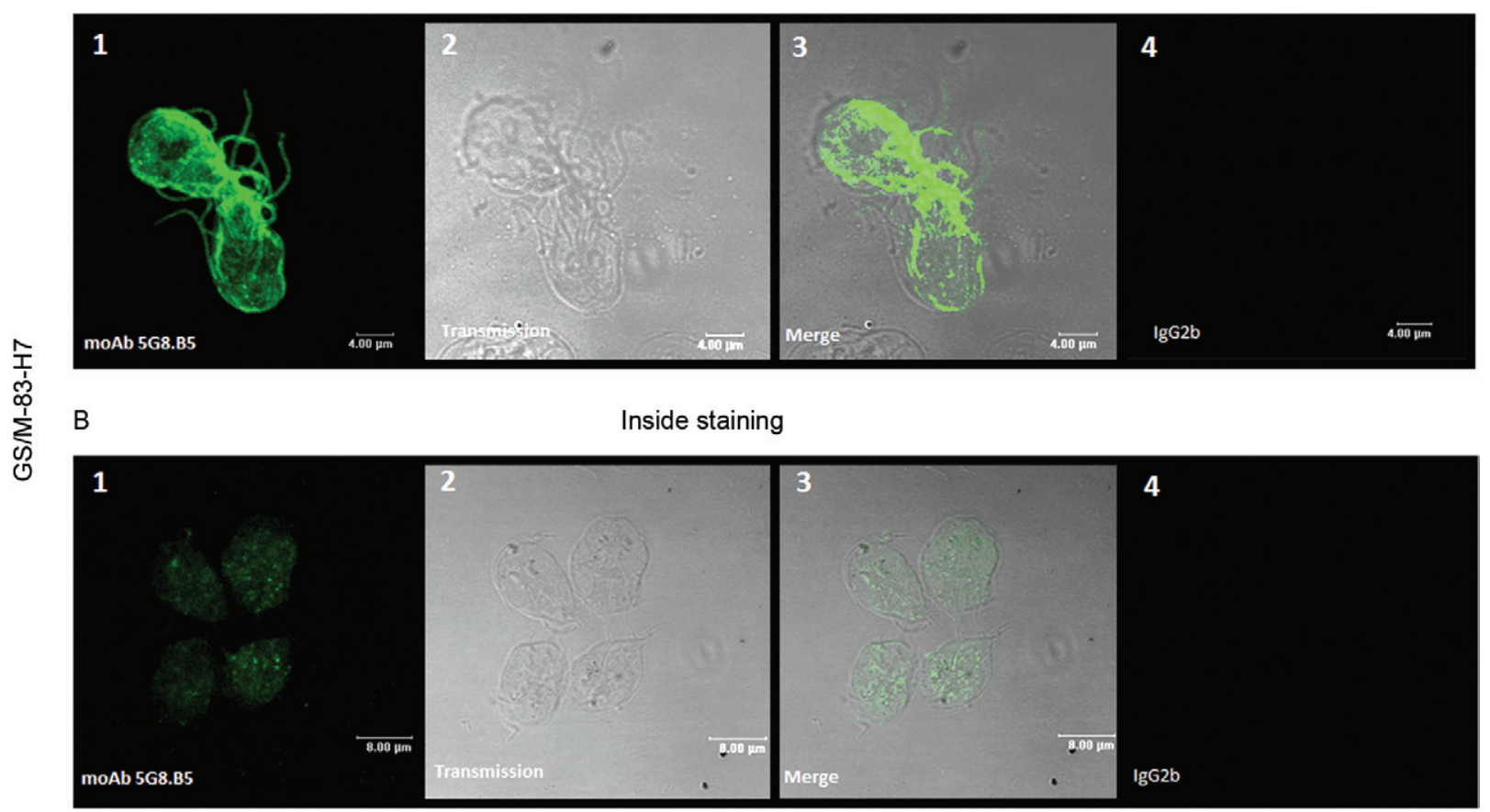

Fig. 4: confocal microscopy analysis of Giardia lamblia trophozoites. Surface (A) and inner (B) staining: 1: 5G8.B5 monoclonal antibody (moAb); 2: transmission microscopy image; 3: merge of 5G8.B5 moAb and transmission microscopy image; 4: isotype control antibody (IgG2b).
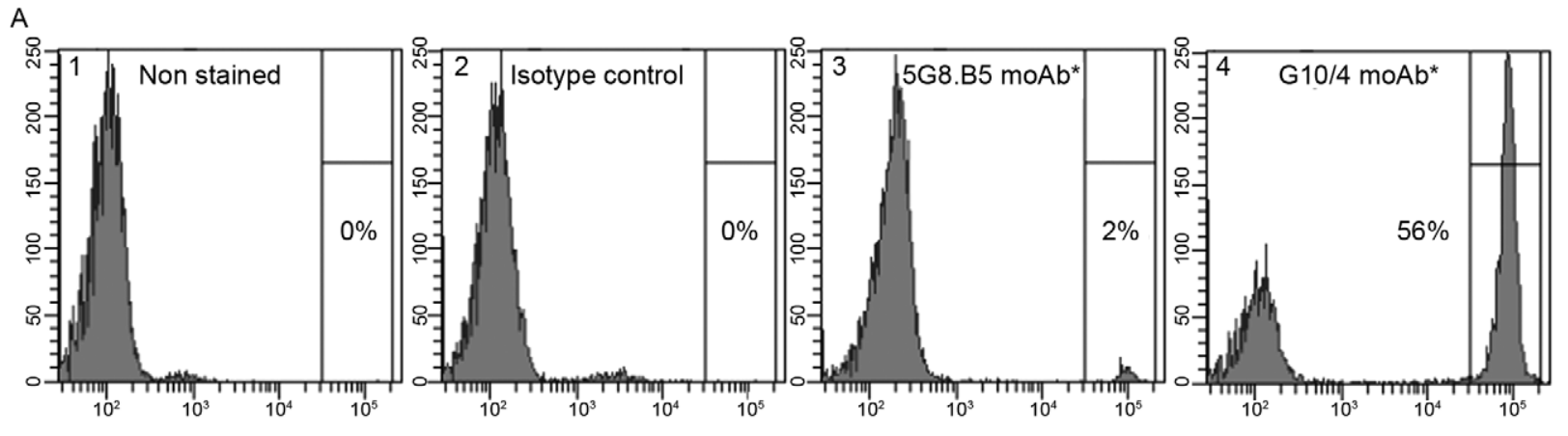

B
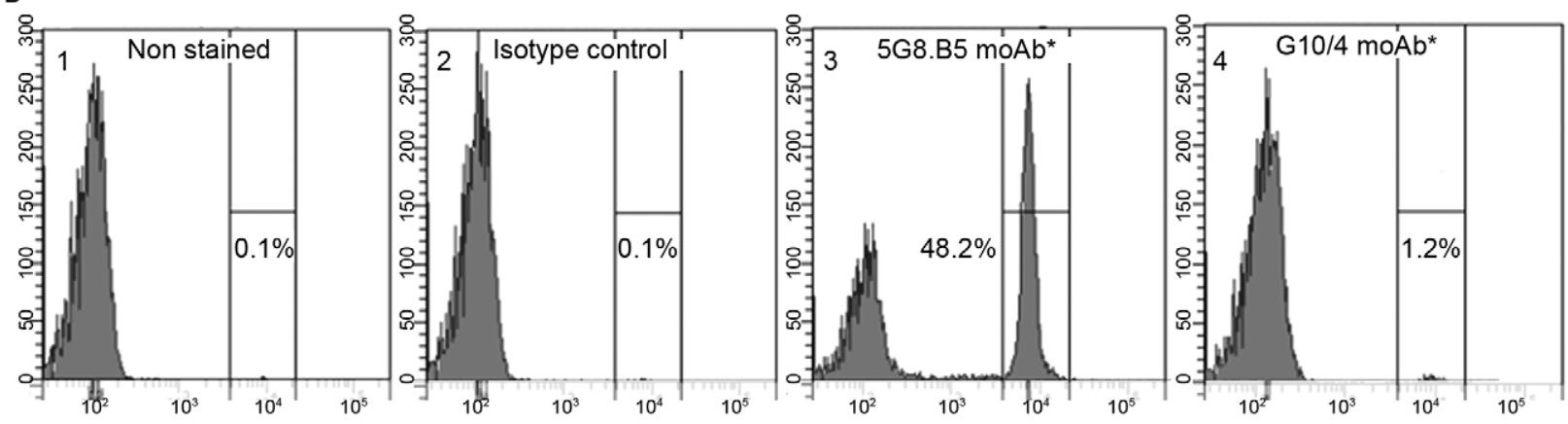

$5 \mathrm{G} 8(+)$ Trophozoites

Fig. 5: Giardia lamblia 5G8 protein is different from variant surface protein (VSP) H7. Fluorescence-activated cell sorting analysis was performed using the monoclonal antibodies (moAbs): G10/4 ( $\alpha$-VSP H7), 5G8.B5 ( $\alpha$-5G8 protein) and isotype control antibody (IgG2b). Cell surface staining of G. lamblia GS/M-83-H7 (A) and 5G8 (+) trophozoites (B). 1: non-stained control cells; 2: isotype control antibody; 3: 5G8.B5 moAb; 4: G10/4 moAb. Statistical significance was accepted when $\mathrm{p}<0.05$ from control antibody (marked with asterisks). 
characterise, at the biochemical and immunological levels, a highly immunogenic protein recognised by the immune system of infected mice. The antigen recognition of most of the moAbs was directed toward a protein band of $\pm 71 \mathrm{kDa}$ (5G8 protein). Primary and secondary infections with $G$. lamblia are associated with differences in the antibody recognition pattern. However, the antibody responses [faecal (IgA) and serum (IgG)] against the 71 $\mathrm{kDa}$ protein were maintained during the primary and secondary infections, demonstrating the immunogenic properties of this antigen (Velazquez et al. 2005). In vitro agglutination of G. lamblia trophozoites was induced by the moAb 5G8.B5, suggesting that in the infected host the 5G8 Giardia protein may induce specific antibodies that could promote Giardia agglutination, thus preventing the colonisation of the parasite. Further studies are needed to characterise this immunogenic protein at the molecular level and evaluate its protective role during

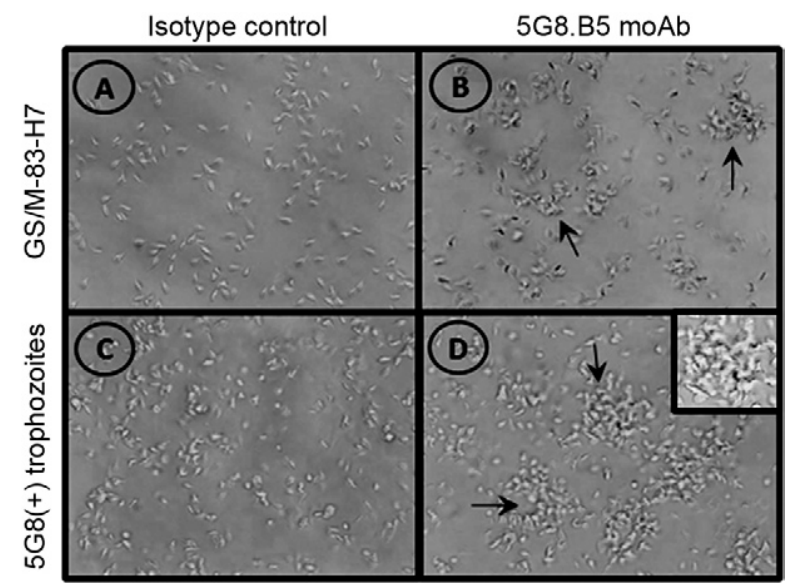

Fig. 6: Giardia lamblia agglutination by the monoclonal antibody (moAb) 5G8.B5. The agglutinating capacity of the 5G8.B5 moAb was evaluated on GS/M-83-H7 and 5G8 (+) trophozoite populations. Control isotype (A, C) and 5G8.B5 (B, D) at different concentrations $(0$, $0.6,1.2,2.5,5$ and $10 \mu \mathrm{g} / \mathrm{mL})$.

\section{TABLE}

Monoclonal antibody 5G8.B5 induces Giardia lamblia trophozoites agglutination ${ }^{a}$

\begin{tabular}{lcc}
$\begin{array}{l}\text { Antibody } \\
(\mu \mathrm{g} / \mathrm{mL})\end{array}$ & Isotype control antibody & moAb 5G8.B5 \\
\hline 0 & - & - \\
0.6 & $+/-$ & + \\
1.2 & $+/-$ & ++ \\
2.5 & $+/-$ & +++ \\
5 & $+/-$ & +++ \\
10 & $+/-$ & ++ \\
\hline
\end{tabular}

a: -: < 1\%; +/-: 1-10\%; +: 11-30\% (low); ++: 31-60\% (medium); +++: 61-90\% (high).
Giardia infection. The G. lamblia genome database ( $G i-$ ardia DB - Giardia Genomic Resources - giardiadb.org/ giardiadb/), together with digestion and mass spectrometry analysis of the antigenic band, may help to identify this G. lamblia antigen (Luque Garcia et al. 2008).

FACS analysis revealed that the 5G8 protein was expressed inside of Giardia trophozoites (>90\% of total cell population) and only a small percentage $(\approx 2-5 \%)$ of those trophozoites expressed high levels of the 5G8 protein on the cell surface. The pattern of expression of this antigen indicates that the 5G8 protein may be transported by the vesicular traffic system toward the plasma membrane (Luján \& Touz 2003). Current studies in our laboratory are examining the precise subcellular localisation of the $5 \mathrm{G} 8$ protein in the trophozoite and its trafficking to the plasma membrane of the parasite.

G. lamblia undergoes antigenic variation, a process resulting in the variation of the proteins that completely cover the trophozoite cell surface. The G. lamblia strain GS/M-83-H7 constitutively expresses the VSP H7, which has a relative molecular mass of $\pm 56 \mathrm{kDa}$, but the migration of this protein in SDS-PAGE is considerably higher $( \pm 72 \mathrm{kDa})$ and can vary with the preparation of the acrylamide gel (Nash \& Mowatt 1992, Luján \& Touz 2003). VSP H7 is expressed on the surface of trophozoites and is immunogenic. The flow cytometry analysis showed a different antibody recognition pattern between the moAbs specific for the proteins 5G8 and VSP H7, indicating that these Giardia molecules are different proteins. In addition, the expected behaviour for a VSP such as VSP H7, which is capable of changing spontaneously in vitro with high frequency (Nash et al. 1990), was observed for VSP H7 protein, but not for the $5 \mathrm{G} 8$ antigen. It has been estimated that the frequency of change (in vitro) for an individual VSP in G. lamblia is approximately once every six-13 generations (48-72 h) (Nash et al. 1990). In contrast, the expression of the 5G8 protein on the surface of G. lamblia trophozoites did not change during in vitro culture for at least three weeks (unpublished observations). This result strongly suggests that the $5 \mathrm{G} 8$ protein is not a VSP.

In summary, this paper describes the identification and partial characterisation of an immunogenic protein of G. lamblia. Additionally, we generated a panel of moAbs specific for this protein, which will be useful for the biochemical and immunological characterisation of this immunologically interesting Giardia molecule.

\section{ACKNOWLEDGEMENTS}

To Theodore Nash and Hugo Lujan, for the donation of the moAb G10/4, and to Bertha Isabel Pacheco, Diana Mendoza Bermudez and Martha Judith Valdez Ortega, for technical assistance.

\section{REFERENCES}

Abdul-Wahid A, Faubert GM 2004. Similarity in cyst wall protein (CWP) trafficking between encysting Giardia duodenalis trophozoites and CWP-expressing human embryonic kidney-293 cells. Biochem Biophys Res Commun 324: 1069-1080.

Adam RD 2001. Biology of Giardia lamblia. Clin Microbiol Rev 14: 447-475. 
Byrd LG, Conrad JT, Nash TE 1994. Giardia lamblia infections in adult mice. Infect Immun 62: 3583-3585.

Davids BJ, Palm JE, Housley MP, Smith JR, Andersen YS, Martin MG, Hendrickson BA, Johansen FE, Svärd SG, Gillin FD, Eckmann L 2006. Polymeric immunoglobulin receptor in intestinal immune defense against the lumen-dwelling protozoan parasite Giardia. J Immunol 177: 6281-6290.

Eckmann L 2003. Mucosal defences against Giardia. Parasite Immunol 25: 259-270.

Eckmann L, Gillin FD 2001. Microbes and microbial toxins: paradigms for microbial-mucosal interactions I. Pathophysiological aspects of enteric infections with the lumen-dwelling protozoan pathogen Giardia lamblia. Am J Physiol Gastrointest Liver Physiol 280: G1-G6.

Faubert G 2000. Immune response to Giardia duodenalis. Clin Microbiol Rev 13: 35-54.

Gottstein B, Harriman GR, Conrad JT, Nash TE 1990. Antigenic variation in Giardia lamblia: cellular and humoral immune response in a mouse model. Parasite Immunol 12: 659-673.

Jenikova G, Hruz P, Andersson MK, Tejman-Yarden N, Ferreira P CD, Andersen YS, Davids BJ, Gillin FD, Svärd SG, Curtiss R 3rd, Eckmann L 2011. A-1 giardin based live heterologous vaccine protects against Giardia lamblia infection in a murine model. Vaccine 29: 9529-9537.

Langford TD, Housley MP, Boes M 2002. Central importance of immunoglobulin A in host defence against Giardia spp. Infect Immun 70: 11-18.

Larocque R, Nakagaki K, Lee P, Abdul-Wahid A, Faubert GM 2003. Oral immunization of BALB/c mice with Giardia duodenalis recombinant cyst wall protein inhibits shedding of cysts. Infect Immun 71: 5662-5669.

Lengerich EJ, Addiss DG, Juranek DD 1994. Severe giardiasis in the United States. Clin Infect Dis 18: 760-763.

Luján HD, Mowatt MR, Conrad JT, Bowers B, Nash TE 1995. Identification of a novel Giardia lamblia cyst wall protein with leucine-rich repeats: implications for secretory granule formation and protein assembly into the cyst wall. J Biol Chem 270: 29307.

Luján HD, Touz MC 2003. Protein trafficking in Giardia lamblia. Cell Microbiol 5: 427-434.

Luque Garcia JL, Zhou Ge, Spellman DS, Sun TT, Neubert TA 2008. Analysis of electroblotted proteins by mass spectrometry: protein identification after western Blotting. Mol Cell Proteomics 7: 308-314.

Nash TE, Banks SM, Alling DW, Merritt Jr JW, Conrado JT 1990. Frequency of variant antigens in Giardia lamblia. Exp Parasitol 71: 415-421.

Nash TE, Mowatt MR 1992. Characterization of a Giardia lamblia variant-specific surface protein (Vsp) gene from isolate $\mathrm{Gs} / \mathrm{M}$ and estimation of the Vsp gene repertoire size. Mol Biochem Parasitol 51: 219-227.

Palm JE, Weiland MEL, Griffiths WJ, Ljungstrom I, Svärd SG 2003. Identification of immunoreactive proteins during acute human giardiasis. J Infect Dis 187: 1849-1859.

Rivero FD, Saura A, Prucca CG, Carranza PG, Torri A, Luján HD 2010. Disruption of antigenic variation is crucial for effective parasite vaccine. Nat Med 16: 551-557.

Roxstrom-Lindquist K, Palm D, Reiner D, Ringqvist E, Svärd SG 2006. Giardia immunity - an update. Trends Parasitol 22: 26-31.

Singer SM, Nash TE 2000. T-cell-dependent control of acute Giardia lamblia infections in mice. Infect Immun 68: 170-175.

Téllez A, Palm D, Weiland M, Almenán J, Winiecka-Krusnell J, Linder E, Svärd S 2005. Secretory antibodies against Giardia intestinalis in lactating Nicaraguan women. Parasite Immunol 27: 163-169.

Touz MC, Rópolo AS, Rivero MR, Vranchy CV, Conrad JT, Svärd SG, Nash TE 2008. Arginine deminase has multiple regulatory roles in the biology of Giardia lamblia. J Cell Sci 121: 2930-2938.

Velazquez C, Beltran M, Ontiveros N, Rascon L, Figueroa DC, Hernandez-Martinez J, Hernandez J, Astiazaran-Garcia H, Granados AJ 2005. Giardia lamblia infection induces different secretory and systemic antibody responses in mice. Parasite Immunol 27 : 351-356. 